\title{
ESMO 2019-personal non-small lung cancer highlights
}

\author{
Lena Horvath · Andreas Pircher
}

Received: 22 January 2020 / Accepted: 25 February 2020 / Published online: 20 March 2020

(C) The Author(s) 2020

\begin{abstract}
Summary This article intends to summarize personal non-small cell lung cancer (NSCLC) highlights of the ESMO 2019 meeting. Again, immunotherapy in the first-line setting of wildtype NSCLC was a major aspect and the search of the optimal biomarker for therapy stratification continues. Moreover, important data on the use of osimertinib, a third-generation epidermal growth factor receptor (EGFR) inhibitor in the first-line setting of EGFR-mutated NSCLC were presented, emerging as the preferred therapeutic strategy in these patients. The ideal treatment sequence, however, remains discussed controversially. The treatment of rare genetic alterations was another important topic, covering updated data on effective NTRK and ROS1 inhibition. In conclusion, ESMO 2019 fueled the lung cancer community with inspiring new data, contributing to a more individualized, hopefully improved lung cancer treatment and continuing to decrease lung cancer mortality.
\end{abstract}

Keywords EGFR · Osimertinib · NTRK · Ipilimumab · Nivolumab · Atezolizumab · NSCLC

\section{Introduction}

ESMO 2019 took place in Barcelona from 27 September to 1 October 2019 and again, treatment of nonsmall cell lung cancer (NSCLC) was a major aspect of the meeting. The congress was held under the tagline "Translating science into better cancer patient care" and intended to bridge basic research and clinical-oncological therapy. With regard to NSCLC, the

\section{Dr. L. Horvath · PD Dr. A. Pircher $(\bowtie)$}

Department of Hematology and Oncology, Internal

Medicine V, Medical University of Innsbruck,

Anichstraße 35, 6020 Innsbruck, Austria

andreas.pircher@i-med.ac.at translational aspect focused on the identification of biomarkers for immunotherapy use and the characterization as well as treatment of druggable genetic alterations. It is becoming apparent that primary or secondary resistance mechanism against immunotherapy as well as targeted therapies can be most likely overcome via rationale combinational therapies.

Due to better treatment options and the plethora of highly effective drugs, the discussion on the right treatment sequence becomes overt and increasingly important to further improve patient care.

\section{Advanced stage non-small cell lung cancer}

\section{Immunotherapy first line without driver alterations}

Two interesting studies (update on CheckMate 227 and IMpower110) were presented, using PD-L1 expression as a biomarker to guide immunotherapy in the first-line setting $[1,2]$.

CheckMate 227 Cohorts of the CheckMate 227 have already been presented at earlier meetings [3, 4] and this presentation focused on the analysis of another co-primary endpoint. In general, the CheckMate 227 is an open-label first-line trial with six cohorts of nivolumab-based regimens compared with standard chemotherapy in patients with stage IV or recurrent NSCLC. The study design is complex and therefore we refer to the main publications for further details [3-5]. Part 1 of the trial comprises 2 cohorts stratified by PD-L1 expression: patients with PD-L1 expression $\geq 1 \%$ (part 1 a) and PD-L1 expression $<1 \%$ (part 1b). In part 1a, 1189 patients were randomized 1:1:1 to receive either histology-based chemotherapy $(n=397)$, nivolumab alone $(n=396)$ or nivolumab plus low-dose ipilimumab $(n=396)$. In part $1 \mathrm{~b}$, 550 patients were randomized to nivolumab/low- 
dose ipilimumab $(n=187)$, chemotherapy $(n=186)$ or nivolumab/chemotherapy $(n=177)$. Nivolumab was administered at $3 \mathrm{mg} / \mathrm{kg}$ every 2 weeks. In the combination arm ipilimumab was given at $1 \mathrm{mg} / \mathrm{kg}$ every 6 weeks. The independent co-primary endpoints of the study focused on comparing nivolumab plus ipilimumab versus chemotherapy and comprised progression-free survival (PFS) in a high tumor mutational burden (TMB) population (i.e., $>10$ mut/Mb) and overall survival (OS) in the PD-L1 $\geq 1 \%$ population. Secondary endpoints included PFS and OS with nivolumab/chemotherapy versus chemotherapy in the PD-L1 $<1 \%$ subgroup, and OS with nivolumab versus chemotherapy in patients with PD-L1 expression $\geq 50 \%$. Data on the co-primary endpoint PFS had already been published before ESMO 2019 and showed a significant PFS benefit for nivolumab plus ipilimumab in patients with a high TMB compared to chemotherapy. However, this observation could not be transferred to final OS data in which PD-L1-high and TMB-high patients showed similar OS results as PD-L1-low and TMB-low patients.

At the current ESMO meeting, Peters et al. [1] presented the OS data of the $\mathrm{PD}-\mathrm{L} 1 \geq 1 \%$ population and the study met its primary endpoint. Median OS was 17.1 versus 14.9 months in the nivolumab/ ipilimumab arm compared to chemotherapy alone (HR for nivolumab/ipilimumab vs. chemotherapy, 0.79; 97.72\% CI, 0.65-0.96). Moreover, median OS was 17.1 months with the combination therapy and 13.9 months with chemotherapy in patients regardless of PD-L1 expression status (HR, 0.73; 95\% CI, 0.64-0.84). Results also showed that in patients with PD-L1 expression $\geq 1 \%$, the 1 - and 2 -year OS rates were 63 and $40 \%$ with nivolumab/ipilimumab and 56 and $33 \%$ with chemotherapy, respectively. The median duration of response (DOR) by blinded independent central review was 23.2 and 6.2 months for nivolumab/ipilimumab and chemotherapy, respectively.

In part $1 \mathrm{~b}$, patients with PD-L1-negative NSCLC also had a numeric advantage of the immune checkpoint inhibitor (ICI) combination, showing a median OS of 17.2 months for nivolumab/ipilimumab versus 12.2 months with chemotherapy (HR for nivolumab/ ipilimumab vs chemotherapy, 0.62; 95\% CI, 0.48-0.78; HR for nivolumab/chemotherapy vs chemotherapy, 0.78; 95\% CI, 0.60-1.02).

In regards of tolerability, no new safety findings for the combination were reported within longer followup. Grade 3/4 treatment-related adverse events were reported in 33 and $36 \%$ of patients in the nivolumab/ ipilimumab versus chemotherapy arms, respectively.

In conclusion, this study could show an OS benefit for the ICI combination therapy compared to chemotherapy, independent of PD-L1 expression. Clinically meaningful benefits were documented in the two subgroups of PD-L1 high $(>50 \%)$ and PD-L1 negative NSCLC. However, clinical utility in the PD-L1 high group is limited as pembrolizumab monotherapy is an already well-established therapy option with more favorable toxicity profile compared to the ICI combo. Although not evaluated as a study endpoint, the advantage of ICI combination in a PD-L1 negative setting is worth being discussed as a good treatment alternative for patients who do not want to be treated with chemotherapy.

IMpower110 A second interesting presentation was the IMpower110 phase III study evaluating atezolizumab as first-line treatment in PD-L1 selected patients with advanced NSCLC, independent of tumor histology. The study enrolled 572 patients with chemotherapy-naïve stage IV NSCLC with PD-L1 expression $\geq 1 \%$ on tumor cells (TC) or immune cells (IC). Patients were randomized 1:1 to receive either atezolizumab $1200 \mathrm{mg}$ every 3 weeks (arm A) or 4 or 6 cycles of platinum-based chemotherapy ( $\operatorname{arm} \mathrm{B}$ ). Primary endpoint of the study was OS by hierarchical clustering, with the analysis of the TC3 or IC3 subgroup preceding evaluation of the TC2/3 or IC2/3 group, which again preceded the $\mathrm{TC} 1 / 2 / 3$ or $\mathrm{IC} 1 / 2 / 3$ group. The TC3 or IC3 population was defined as PDL1 expression $\geq 50 \%$ on tumor cells (TC3) or $\geq 10 \%$ on tumor-infiltrating immune cells (IC3).

At ESMO 2019 D. Spigel [2] presented an interim OS analysis of the TC3 and IC3 subgroup, showing an improved OS for atezolizumab monotherapy compared to platinum-based chemotherapy as a first-line treatment in patients with wild-type NSCLC. At a median follow-up of 15.7 months (range, 0-35 months), median OS was 20.2 months (95\% CI, 16.5-not evaluable) for the atezolizumab arm, compared to 13.1 months (95\% CI, 7.4-16.5) in the chemotherapy arm (HR, 0.59; 95\% CI, 0.40-0.89; $P=0.0106)$. The OS testing boundary was not crossed in the TC2/3 or IC2/3 wild-type population; therefore, OS was not formally tested in this population as well as in the $\mathrm{TC} 1 / 2 / 3$ and $\mathrm{IC} 1 / 2 / 3$ populations. Median PFS was 8.1 months (95\% CI, 6.8-11.0) in the atezolizumab arm versus 5.0 months (95\% CI, 4.2-5.7) in the chemotherapy arm (HR, 0.63; 95\% CI, 0.45-0.88; $P=0.007$ ) in the TC3/IC3 wild-type population; the confirmed ORR was $38.3 \%$ vs. $28.6 \%$, respectively; the median DOR was not reached versus 6.7 months, respectively.

The authors of the study concluded that atezolizumab represents a promising first-line treatment option in NSCLC patients with high PD-L1 expression. Interestingly, this study showed for the first time that PD-L1 expression on immune cells plays an important role in therapy stratification and can also be used as predictive biomarker. However, we have to await how these data will be integrated into our common treatment approach of PD-L1 high patients. Lastly, IMpower 110 could show the applicability of the less commonly used PD-L1 score TC3/IC3 (IHC antibody Ventana SP142) in this patient setting. Due to the widespread use of the PD-L1 TPS score (IHC antibody 
Dako 22C3) it would be beneficial for clinical practice to validate the study results with the latter biomarker.

\section{Targeted therapies in genetically driven NSCLC}

Frontline EGFR inhibition represents the gold standard therapy in EGFR-mutated NSCLC and up to now three different generations of EGFR inhibitors have been approved.

The FLAURA study is a landmark study [6], which proved that the third generation irreversible EGFR-tyrosine kinase inhibitor (TKI) osimertinib significantly prolonged PFS compared to first generation EGFR-TKI erlotinib or gefitinib (18.9 months versus 10.2 months; HR 0.42). At the first FLAURA presentation 2018, OS data were not yet mature and at ESMO 2019 S. Ramalingam presented the final OS results [7]. FLAURA could translate the PFS benefit into a significant OS benefit (38.6 months versus 31.8 months; HR 0.79), proving clinically meaningful OS benefit for the first time in this setting. As OS was a secondary endpoint of the study it was not powered for all subgroups. However, most subgroups gained OS benefit from osimertinib compared to erlotinib and gefitinib.

Regarding subsequent therapies after disease progression, around $30 \%$ of patients did not receive a second-line therapy in either arm. The predominant second-line therapy was osimertinib (47\%) in the control arm and cytotoxic therapy (68\%) in the osimertinib arm. The toxicity profile showed no new signs over time and good tolerance.

In conclusion, FLAURA shows that osimertinib is the preferred agent in the first-line setting of EGFRmutated NSCLC. Nevertheless, the optimal treatment sequence and other questions, such as synergistic combinational partners, remain open and controversially discussed.

In the setting of rare mutations, updated data on the subgroups of NTRK-positive and ROS1-rearranged NSCLC were presented [8].

In NTRK-positive NSCLC $(n=10)$, entrectinib showed an ORR of $70 \%$ with a complete response rate (CRR) of $10 \%$ and a partial response rate (PRR) of $60 \%$. In ROS1-positive NSCLC patients entrectinib showed an ORR of $79.2 \%$ with an CRR of $9.4 \%$. The median DOR was 24.6 months (95\% CI 12.6-34.8), median PFS was 19.0 months (95\% CI 12.2-29.6), and median OS was not estimable (95\% CI 30.8-NE). These results underline the high efficacy of entrectinib in NTRK-positive and ROS1-rearranged NSCLC. Furthermore, these studies highlight the significance of broad molecular testing in NSCLC patients and that patients with molecular alterations should be treated with specific inhibitors as response rates are high and long-lasting. Nevertheless, long-term efficacy has to be proven and the optimal sequence has to be established.

\section{Take-home messages}

- Nivolumab/lpilimumab combination improves OS compared to chemotherapy independent of PD-L1 status and presents a potential chemotherapy-free option as first-line treatment of selected NSCLC IV patients.

- Atezolizumab improves OS in PD-L1 high wildtype NSCLC in the first-line setting compared to chemotherapy.

- Osimertinib prolongs OS in EGFR-mutated NSCLC IV and evolves as new first-line treatment standard.

- Entrectinib shows high efficacy in NTRK and ROS1mutated NSCLC. Mutational screening should be warranted.

Funding The review was supported by the "Verein für Tumorfoschung", Anichstraße 35, Innsbruck, Austria.

Funding Open access funding provided by University of Innsbruck and Medical University of Innsbruck.

Conflict of interest L. Horvath has no conflicts of interest to declare. A. Pircher has received speaker's fees and honoraria for advisory boards from Astra Zeneca, BMS, Roche, Pfizer, Takeda and MSD.

Open Access This article is licensed under a Creative Commons Attribution 4.0 International License, which permits use, sharing, adaptation, distribution and reproduction in any medium or format, as long as you give appropriate credit to the original author(s) and the source, provide a link to the Creative Commons licence, and indicate if changes were made. The images or other third party material in this article are included in the article's Creative Commons licence, unless indicated otherwise in a credit line to the material. If material is not included in the article's Creative Commons licence and your intended use is not permitted by statutory regulation or exceeds the permitted use, you will need to obtain permission directly from the copyright holder. To view a copy of this licence, visit http://creativecommons.org/licenses/by/4.0/.

\section{References}

1. Peters S, Ramalingam SS, Paz-Ares L, et al. LBA4_PRNivolumab (NIVO) + low-dose ipilimumab (IPI) vs platinumdoublet chemotherapy (chemo) as first-line (1L) treatment (tx) for advanced non-small cell lung cancer (NSCLC): CheckMate 227 part 1 final analysis. Ann Oncol. 2019;30:v851-v934. https://doi.org/10.1093/annonc/ mdz394

2. SpigelD, de Marinis F, GiacconeG, etal. LBA78IMpower110: Interim overall survival (OS) analysis of a phase III study of atezolizumab (atezo) vs platinum-based chemotherapy (chemo) as first-line (1L) treatment (tx) in PD-L1-selected NSCLC. Ann Oncol. 2019;30:v851-v934. https://doi.org/ 10.1093/annonc/mdz394

3. Borghaei H, Hellmann MD, Paz-Ares LG, et al. Nivolumab (Nivo) + platinum-doublet chemotherapy (Chemo) vs chemo as first-line (1L) treatment (Tx) for advanced nonsmall cell lung cancer (NSCLC) with $\langle 1 \%$ tumor PD-L1 expression: Results from CheckMate 227. J Clin Oncol. 2018;36:9001-9001. 
4. Hellmann MD, Ciuleanu TE, Pluzanski A, et al. Nivolumab plus Ipilimumab in lung cancer with a high tumor mutational burden. NEngl J Med. 2018;378:2093-104.

5. Hellmann MD, Paz-Ares L, BernabeCaro R, et al. Nivolumab plus ipilimumab in advanced non-small-cell lung cancer. NEngl J Med. 2019;381:2020-31.

6. Soria JC, Ohe Y, Vansteenkiste J, et al. Osimertinib in untreated EGFR-mutated advanced non-small-cell lung cancer. NEngl J Med. 2018;378:113-25.

7. Ramalingam SS, Vansteenkiste J, Planchard D, et al. Overall survival with osimertinib in untreated. N Engl J Med. 2020;382:41-50.

8. de Braud F, Siena S, Barlesi F, et al. 64PEntrectinib in locally advanced/metastatic ROS1 and NTRKfusion-positive non-small cell lung cancer (NSCLC): Updated integrated analysis of STARTRK-2, STARTRK-1 and ALKA-372-001.
Ann Oncol. 2019;30:v602-v660. https://doi.org/10.1093/ annonc/mdz260

Publisher's Note Springer Nature remains neutral with regard to jurisdictional claims in published maps and institutional affiliations.

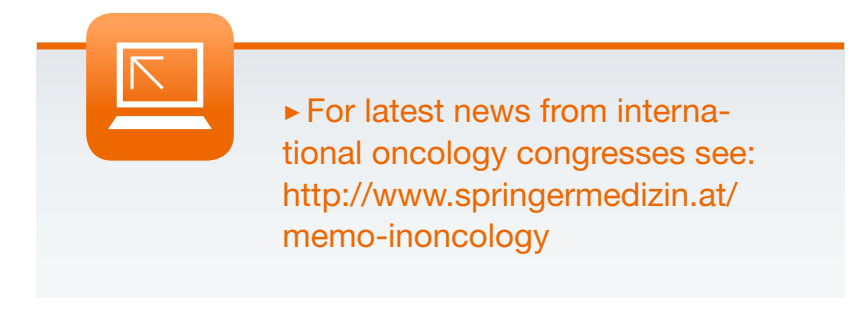

